\title{
STABILITY OF A CLASS OF HYBRID IMPULSIVE AND SWITCHING SYSTEMS ${ }^{1}$
}

\author{
Zhi-Hong Guan*, David J. Hill**, \\ Xuemin (Sherman) Shen ${ }^{* * *}$
}

\author{
* Dept. of Control Science and Engineering \\ Huazhong University of Science and Technology \\ Wuhan, 430074,P.R. China.zhguan@mail.hust.edu.cn \\ ** Dept. Information Eng., The Australian National Univ. \\ CanberraACT0200 Australia.david.hill@anu.edu.au \\ ${ }^{* * *}$ Dept. Elec. and Comp. Eng., Univ. of Waterloo \\ Ontario, Canada N2L3G1.xshen@bbcr.uwaterloo.ca
}

\begin{abstract}
In this paper, a new class of hybrid impulsive and switching models are introduced. Using switched Lyapunov functions, some new general criteria for exponential stability and asymptotic stability of hybrid impulsive and switched nonlinear systems are established. Further more, a new hybrid impulsive and switching control strategy for the nonlinear systems is developed to improve the system performance. A typical example, the Chua's chaotic circuit, is provided for illustrating and visualizing the analytical results. Copyright ${ }^{(C)} 2005$ IFAC
\end{abstract}

Keywords: Hybrid systems, Impulsive control, Switching systems, Switched Lyapunov function, Exponential stability, Chaos control

\section{INTRODUCTION}

Hybrid systems consisting of interacting continuous and discrete dynamics under certain logic rules, have gained considerable attention in science and engineering (Aubin et al., 2001; Branicky, 1998; Decarlo et al., 2000; Ge et al., 2001; Hespanha and Morse, 1999; Li et al., 2001; Liberzon and Morse, 1999; Mancilla-Aguilar, 2003), since they provide a natural and convenient unified framework for mathematical modeling of many complex physical phenomena and practical applications. Examples include robotics, integrated circuit design, multi-media, manufac-

\footnotetext{
1 This work was supported by the National Natural Science Foundation of China under Grants 60074009 and 60274004, Hong Kong RGC under CERG 1232/02E, and City University of Hong Kong Project No. 9380026.
}

turing, power electronics, switched-capacitor networks, chaos generators, automated highway systems and air traffic management systems. So far, most research work on hybrid systems has been devoted to stability analysis and stabilization, (Decarlo et al., 2000; Liberzon and Morse, 1999; Liberzon, 2003; Michel, 1999). Most recently, on the basis of Lyapunov functions and other analysis tools, the stability and stabilization for linear or nonlinear switched systems have been further investigated and many valuable results have been obtained (Daafouz et al., 2002; Ge et al., 2001; Ishii and Francis, 2002; Leonessa et al., 2001; Li et al., 2001; Mancilla-Aguilar, 2003), etc..

In general, switching systems can be classified into two groups: continuous and discrete transition switching systems (Liberzon, 2003). However, 
there are still many switching systems existing in the real world, which display a certain kind of dynamics with impulse effect at the switching points. Examples of these systems include many evolutionary processes, particularly some biological systems such as biological neural networks and bursting rhythm models in pathology. Other examples include optimal control models in economics, frequency-modulated signal processing systems, and flying object motions. Moreover, impulsive and switching phenomena can also be found in the fields of information science, electronics, automatic control systems, computer networking, artificial intelligence, robotics, and telecommunications. All these systems are characterized by switches of states and abrupt changes at the switching instants, that is, the systems switch with impulse effect (Bainov and Simeonov, 1989; Lakshmikantham et al., 1999; Liu and Guan, 1996). Many sudden and sharp changes occur instantaneously, in the form of impulses and switches, which cannot be well described by using pure continuous or pure discrete models. Therefore, it is very important and necessary to study impulsive and switching systems. This motivates the present investigation of hybrid impulsive and switching nonlinear systems.

In this paper, the exponential and asymptotic stabilities of hybrid impulsive and switching nonlinear systems are investigated by using switched Lyapunov functions, and some new general stability criteria are established. The rest of the paper is organized as follows. In section 2, an analytical model is formulated for the hybrid nonlinear impulsive and switching systems. Based on the model, the exponential and asymptotic stabilities are investigated. Numerical example is given in section 4 to verify the analytical results, followed by the conclusions in section 5 .

\section{PROBLEM FORMULATION}

Let $R_{+}=[0,+\infty), J=\left[t_{0},+\infty\right)\left(t_{0} \geq 0\right)$, $R^{n}$ denote the $n$-dimensional Euclidean space. For $x=\left(x_{1}, \ldots, x_{n}\right)^{\top} \in R^{n}$, the norm of $x$ is $\|x\|:=\left(\sum_{i=1}^{n} x_{i}^{2}\right)^{1 / 2}$. For $A=\left(a_{i j}\right)_{n \times n} \in R^{n \times n}$, $\lambda_{\max }(A)$ and $\lambda_{\min }(A)$ are the maximal and the minimum eigenvalue of $A$, respectively. The identity matrix of order $m$ is denoted as $I_{m}$ (or simply $I$ if no confusion arises).

Consider the hybrid impulsive and switching system with the following form:

$$
\begin{cases}\dot{x}=A_{i_{k}} x+F_{i_{k}}(t, x), & t \in\left(t_{k-1}, t_{k}\right] \\ \triangle x=B_{k} x, & t=t_{k} \\ x\left(t_{0}^{+}\right)=x_{0}, & k=1,2, \cdots,\end{cases}
$$

where $t \in J, x \in R^{n}$ is the state variable, $A_{i_{k}}$ and $B_{k}$ are $n \times n$ matrices, $i_{k} \in\{1,2, \cdots, m\}$ is the switching index, the time sequence $\left\{t_{k}\right\}$ satisfies

$$
t_{1}<t_{2}<\cdots<t_{k}<\cdots, \quad \lim _{k \rightarrow \infty} t_{k}=\infty
$$

where $t_{1}>t_{0},\left.\triangle x\right|_{t=t_{k}}=x\left(t_{k}^{+}\right)-x\left(t_{k}\right)$, and $F_{i_{k}}(t, x): J \times R^{n} \longmapsto R^{n}$ are piecewise continuous vector-value functions with $F_{i_{k}}(t, 0) \equiv 0, t \in J$.

It is obvious that, system (1) has $m$ different modes, that is,

$$
\dot{x}=A_{i} x+F_{i}(t, x), \quad i=1,2, \cdots, m,
$$

switching in the interval $J$. For any $t \in J$, let $T_{i}\left(t_{0}, t\right)$ be the union of the small switching intervals included in $\left[t_{0}, t\right]$, on which, the corresponding $i$ th subsystem (2) is activated. Then the first equation of system (1) can be rewritten as

$$
\dot{x}=A_{i} x+F_{i}(s, x), \quad s \in T_{i}\left(t_{0}, t\right), \quad t \in J,
$$

where $i=1,2, \cdots, m, \bigcup_{i=1}^{m} T_{i}\left(t_{0}, t\right)=\left[t_{0}, t\right]$.

System (1), in some sense, is a general framework for nonlinear hybrid models, which includes some well-known models as its special cases. For instance, in system (1), if $B_{k} \equiv 0$, then it turns into

$$
\dot{x}=A_{i_{k}} x+F_{i_{k}}(t, x), \quad t \in\left(t_{k-1}, t_{k}\right],
$$

with $i_{k} \in\{1,2, \cdots, m\}$, which is a typical nonlinear switching system. Stability of the systems, similar to system (4), has been investigated (Decarlo et al., 2000; Liberzon and Morse, 1999; Liberzon, 2003; Michel, 1999).

Similarly, if $m=1$, the system (1) becomes

$$
\begin{cases}\dot{x}=A x+F(t, x), & t \in\left(t_{k-1}, t_{k}\right] \\ \triangle x=B_{k} x, & t=t_{k} \\ x\left(t_{0}^{+}\right)=x_{0}, & k=1,2, \cdots,\end{cases}
$$

which is a typical nonlinear impulsive system. Stability of system (5) has been extensively studied (e.g., (Bainov and Simeonov, 1989; Guan et al., 2000; Khadra et al., 2003; Lakshmikantham et al., 1999; Liu and Guan, 1996) and some references therein) .

Also, if $B_{k} \equiv 0$ and $m=1$, the system (1) reduces to a general nonlinear system

$$
\dot{x}=A x+F(t, x) \text {. }
$$

A typical characteristic of the nonlinear hybrid system (1) that differs from most existing models (see, surveys (Decarlo et al., 2000; Liberzon and Morse, 1999; Liberzon, 2003; Michel, 1999) and the references therein) is its discontinuity in the form of impulses and switches. Therefore, to ensure that it can be successfully used to describe and to deal with various impulsive and switching phenomena, especially some evolution processes involving impulses and switches in the real world (Bainov and Simeonov, 1989; Lakshmikantham et 
$a l ., 1999)$, a detailed investigation of this new model is essential.

In what follows, the globally asymptotic and exponential stability of the hybrid model (1) is studied.

\section{STABILITY ANALYSIS}

Lemma 3.1. If $P \in R^{n \times n}$ is a positive definite matrix, $Q \in R^{n \times n}$ is a symmetric matrix, then

$\lambda_{\min }\left(P^{-1} Q\right) x^{\top} P x \leq x^{\top} Q x \leq \lambda_{\max }\left(P^{-1} Q\right) x^{\top} P x$ for any $x \in R^{n}$.

Lemma 3.2. If $P \in R^{n \times n}$ is a positive definite matrix, $F(t, x) \in R^{n}$ and $\|F(t, x)\| \leq L(t)\|x\|$, where $x \in R^{n}, t \in J$, and $L(t) \geq 0$, then

$$
F^{\top}(t, x) P x \leq\left[\lambda_{\max }(P) / \lambda_{\min }(P)\right]^{\frac{1}{2}} L(t) x^{\top} P x .
$$

For the asymptotic properties of the hybrid system (1), assume that, for $t \in J, x \in R^{n}$, there exist continuous functions $\varphi_{i}(t) \geq 0$ and positive definite matrices $P_{i}$, such that

$$
F_{i}^{\top}(t, x) P_{i} x \leq \varphi_{i}(t) x^{\top} P_{i} x, \quad i=1,2, \cdots, m .
$$

Furthermore, for convenience, define the following functions $\lambda_{i}(t)$ and parameters $\beta_{k}$ and $\rho$ by inequalities and equalities:

$$
\begin{gathered}
\lambda_{\max }\left[P_{i}^{-1}\left(A_{i}^{\top} P_{i}+P_{i} A_{i}\right)\right]+2 \varphi_{i}(t) \leq \lambda_{i}(t), \\
\lambda_{\max }\left[\left(I+B_{k}\right)^{\top}\left(I+B_{k}\right)\right] \leq \beta_{k}, \\
\rho=\max _{1 \leq i \leq m}\left\{\rho_{i}^{2}\right\}, \quad \rho_{i}=\left[\lambda_{\max }(P) / \lambda_{\min }(P)\right]^{\frac{1}{2}}
\end{gathered}
$$

where $i=1,2, \cdots, m, k=1,2, \cdots$.

Remark 3.1. In general, for nonlinear functions $F_{i}(t, x)$, one has the Lipschitz assumption, that is, for $x \in R^{n}, t \in J$, there exist continuous functions $L_{i}(t) \geq 0$ satisfying

$$
\left\|F_{i}(t, x)\right\| \leq L_{i}(t)\|x\|, \quad i=1,2, \cdots, m .
$$

This immediately implies from Lemma 3.2 that the Lipschitz assumption is a special case of (6).

Theorem 3.1. (i) If $\lambda_{i}(t) \leq-\lambda_{i}<0, \lambda_{i}>0$ are constants, $i=1,2, \cdots, m$, and there exists a constant $0<\alpha<\lambda_{i}, i=1,2, \cdots, m$, such that

$$
\ln \left(\rho \beta_{k}\right)-\alpha\left(t_{k}-t_{k-1}\right) \leq 0, k=1,2, \cdots
$$

then the trivial solution of system (1) is globally exponential stable, where $\lambda_{i}(t), \beta_{k}$, and $\rho$ are given by (7), (8), and (9), respectively.

(ii) If $0 \leq \lambda_{i}(t) \leq \lambda(t), i=1,2, \cdots, m$, and there exists a constant $\alpha \geq 1$ such that

$$
\ln \left(\alpha \rho \beta_{k}\right)+\int_{t_{k}}^{t_{k+1}} \lambda(s) d s \leq 0, k=1,2, \cdots
$$

then $\alpha=1$ implies that the trivial solution of system (1) is stable and $\alpha>1$ implies that the trivial solution of system (1) is globally asymptotical stable, where $\lambda_{i}(t), \beta_{k}$, and $\rho$ are given by $(7),(8)$, and $(9)$, respectively.

Proof. Construct the switched Lyapunov function in the form of

$$
V_{i_{k}}(x)=x^{\top} P_{i_{k}} x, \quad i_{k} \in\{1,2, \cdots, m\},
$$

where $P_{i_{k}}$ is a positive definite matrix given by (6), and let $V_{i_{k}}(t)=: V_{i_{k}}(x(t))$. Since Eqs. (6) and (7) hold, from Lemma 3.1, the total derivative of $V_{i_{k}}(x)$, with respect to $(1)$, is

$$
\begin{aligned}
& \left.\dot{V}_{i_{k}}(x(t))\right|_{(1)} \\
= & {\left[A_{i_{k}} x+F_{i_{k}}(t, x)\right]^{\top} P_{i_{k}} x+x^{\top} P_{i_{k}}\left[A_{i_{k}} x\right.} \\
& \left.+F_{i_{k}}(t, x)\right] \\
= & x^{\top}\left[A_{i_{k}}^{\top} P_{i_{k}}+P_{i_{k}} A_{i_{k}}\right] x+2 F_{i_{k}}^{\top}(t, x) P_{i_{k}} x \\
\leq & \left\{\lambda_{\max }\left[P_{i_{k}}^{-1}\left(A_{i_{k}}^{\top} P_{i_{k}}+P_{i_{k}} A_{i_{k}}\right)\right]+2 \varphi_{i}(t)\right\} . \\
& x^{\top} P_{i_{k}} x \\
\leq & \lambda_{i_{k}}(t) V_{i_{k}}(t), \quad t \in\left(t_{k-1}, t_{k}\right],
\end{aligned}
$$

which implies that, for $t \in\left(t_{k-1}, t_{k}\right]$,

$$
V_{i_{k}}(t) \leq V_{i_{k}}\left(t_{k-1}^{+}\right) \exp \left[\int_{t_{k-1}}^{t} \lambda_{i_{k}}(s) d s\right],
$$

where $k=1,2, \cdots, \lambda_{i_{k}}(t)$ is given by Eq. (7). It immediately follows from (13) that,

$$
\begin{aligned}
x^{\top}(t) P_{i_{k}} x(t) \leq & x^{\top}\left(t_{k-1}^{+}\right) P_{i_{k}} x\left(t_{k-1}^{+}\right) \\
& \times \exp \left[\int_{t_{k-1}}^{t} \lambda_{i_{k}}(s) d s\right], t \in\left(t_{k-1}, t_{k}\right],
\end{aligned}
$$

which leads to

$$
\begin{aligned}
& \lambda_{\min }\left(P_{i_{k}}\right) x^{\top}(t) x(t) \\
\leq & \lambda_{\max }\left(P_{i_{k}}\right) x^{\top}\left(t_{k-1}^{+}\right) x\left(t_{k-1}^{+}\right) \exp \left[\int_{t_{k-1}}^{t} \lambda_{i_{k}}(s) d s\right],
\end{aligned}
$$

or,

$$
w(t) \leq \rho w\left(t_{k-1}^{+}\right) \exp \left[\int_{t_{k-1}}^{t} \lambda_{i_{k}}(s) d s\right],
$$

where $t \in\left(t_{k-1}, t_{k}\right], \rho$ is defined in $(9)$, and

$$
w(t)=x^{\top}(t) x(t) .
$$

On the other hand, it follows from (1) that 


$$
\begin{aligned}
w\left(t_{k}^{+}\right) & =\left[\left(I+B_{k}\right) x\left(t_{k}\right)\right]^{\top}\left(I+B_{k}\right) x\left(t_{k}\right) \\
& \leq \lambda_{\max }\left[\left(I+B_{k}\right)^{\top}\left(I+B_{k}\right)\right] x^{\top}\left(t_{k}\right) x\left(t_{k}\right) \\
& \leq \beta_{k} w\left(t_{k}\right), \quad k=1,2, \cdots,
\end{aligned}
$$

where $\beta_{k} \geq 0$ is given by Eq. (8).

By Eqs. (14) and (16), it is easy to get that, for $t \in\left(t_{k}, t_{k+1}\right]$,

$$
\begin{aligned}
w(t) & \leq w\left(t_{0}^{+}\right) \rho^{k+1} \beta_{1} \cdots \beta_{k} \exp \left[\int_{t_{0}}^{t_{1}} \lambda_{i_{1}}(s) d s\right. \\
& \left.+\int_{t_{1}}^{t_{2}} \lambda_{i_{2}}(s) d s+\cdots+\int_{t_{k}}^{t} \lambda_{i_{k+1}}(s) d s\right] .
\end{aligned}
$$

(i) When $\lambda_{i}(t) \leq-\lambda_{i}<0, i=1,2, \cdots, m$, let $\lambda=\min _{1 \leq i \leq m}\left\{\lambda_{i}\right\}$, it follows from (16) and (17) that

$$
\begin{aligned}
& w(t) \leq w\left(t_{0}^{+}\right) \rho^{k+1} \beta_{1} \cdots \beta_{k} \times \\
& e^{-\lambda_{i_{1}}\left(t_{1}-t_{0}\right)-\lambda_{i_{2}}\left(t_{2}-t_{1}\right)-\cdots-\lambda_{i_{k}}\left(t_{k}-t_{k-1}\right)-\lambda_{i_{k+1}}\left(t-t_{k}\right)} \\
& \leq w\left(t_{0}^{+}\right) \rho^{k+1} \beta_{1} \cdots \beta_{k} e^{-\lambda\left(t-t_{0}\right)} \\
&=w\left(t_{0}^{+}\right) \rho^{k+1} \beta_{1} \cdots \beta_{k} e^{-\alpha\left(t-t_{0}\right)} e^{-(\lambda-\alpha)\left(t-t_{0}\right)} \\
& \leq w\left(t_{0}^{+}\right) \rho^{k+1} \beta_{1} \cdots \beta_{k} e^{-\alpha\left(t_{k}-t_{0}\right)} e^{-(\lambda-\alpha)\left(t-t_{0}\right)} \\
&=w\left(t_{0}^{+}\right) \rho \beta_{1} e^{-\alpha\left(t_{1}-t_{0}\right)} \cdots \\
& \times \rho \beta_{k} e^{-\alpha\left(t_{k}-t_{k-1}\right)} \rho e^{-(\lambda-\alpha)\left(t-t_{0}\right)} \\
& \leq w\left(t_{0}^{+}\right) \rho e^{-(\lambda-\alpha)\left(t-t_{0}\right)}, t \in\left(t_{k}, t_{k+1}\right],
\end{aligned}
$$

namely,

$$
w(t) \leq w\left(t_{0}^{+}\right) \rho e^{-(\lambda-\alpha)\left(t-t_{0}\right)}, t \geq t_{0},
$$

which implies that the trivial solution of system (1) is globally exponential stable.

(ii) When $0 \leq \lambda_{i}(t) \leq \lambda(t), i=1,2, \cdots, m$, it follows from (11) and (17) that, for $t \in\left(t_{k}, t_{k+1}\right]$,

$$
\begin{aligned}
w(t) & \leq w\left(t_{0}^{+}\right) \rho^{k+1} \beta_{1} \cdots \beta_{k} \exp \left[\int_{t_{0}}^{t_{1}} \lambda_{i_{1}}(s) d s\right. \\
& \left.+\int_{t_{1}}^{t_{2}} \lambda_{i_{2}}(s) d s+\cdots+\int_{t_{k}}^{t_{k+1}} \lambda_{i_{k+1}}(s) d s\right] \\
& =w\left(t_{0}^{+}\right) \frac{\rho}{\alpha^{k}} \exp \left[\int_{t_{0}}^{t_{1}} \lambda(s) d s\right] \alpha \rho \beta_{1} \\
& \times \exp \left[\int_{t_{1}}^{t_{2}} \lambda(s) d s\right] \cdots \alpha \rho \beta_{k} \exp \left[\int_{t_{k}}^{t_{k+1}} \lambda(s) d s\right] \\
& \leq w\left(t_{0}^{+}\right) \frac{\rho}{\alpha^{k}} \exp \left[\int_{t_{0}}^{t_{1}} \lambda(s) d s\right],
\end{aligned}
$$

which implies that the conclusion (ii) of Theorem 3.1 holds. This completes the proof.

Remark 3.2. In the case of (ii) in Theorem 3.1, the condition $\lambda_{i}(t) \geq 0$ implies that the stability for switching subsystem (2) is not necessary, that is, each switching subsystem $\dot{x}=A_{i} x+F_{i}(t, x)$ may be unstable, but the corresponding hybrid impulsive and switching system (1) is asymptotically stable.

It is observed that, the $\lambda_{1}(t), \lambda_{2}(t), \cdots, \lambda_{m}(t)$ may not be all positive or negative in small switching intervals. To cover this situation, the asymptotic properties of the hybrid system (1) are further considered.

Theorem 3.2. Assume that the impulsive switching of system (1) satisfies $\beta_{k} \leq \beta, k=1,2, \cdots$, and

$$
\sum_{i=1}^{m} \int_{T_{i}\left(t_{0}, t\right)} \lambda_{i}(s) d s \leq \psi\left(t_{0}, t\right), \quad t \geq t_{0},
$$

where $T_{i}\left(t_{0}, t\right)$ is defined in $(3), \psi\left(t_{0}, t\right)$ is a function on $J, \lambda_{i}(t)$ and $\beta_{k}$ are given by (7) and (8), respectively, then the following conclusions hold.

(i) If $\rho \beta \leq 1, \rho$ is defined by (8), then

$$
\lim _{t \rightarrow+\infty} \psi\left(t_{0}, t\right)=-\infty
$$

implies that the trivial solution of system (1) is globally asymptotical stable, and

$$
\psi\left(t_{0}, t\right) \leq-c\left(t-t_{0}\right), \quad t \geq t_{0}
$$

with $c>0$ being constant, implies that the trivial solution of system (1) is globally exponential stable.

(ii) If $\rho \beta>1, \rho$ is defined by (8), $t_{k}-t_{k-1} \geq \delta>$ $0, k=1,2, \cdots$, then

$\lim _{t \rightarrow+\infty}\left[\frac{\ln (\rho \beta)}{\delta}\left(t-t_{0}\right)+\psi\left(t_{0}, t\right)\right]=-\infty$

implies that the trivial solution of system (1) is globally asymptotically stable, and

$$
\frac{\ln (\rho \beta)}{\delta}\left(t-t_{0}\right)+\psi\left(t_{0}, t\right) \leq-c\left(t-t_{0}\right),
$$

with $t \geq t_{0}, c>0$ being constant, implies that the trivial solution of system (1) is globally exponentially stable.

Remark 3.3. In Theorems 3.1-3.2, if we choose all the switching intervals to be equivalent, that is, $t_{k}-t_{k-1}=\tau=$ constant, $k=1,2, \cdots$, then the corresponding conditions are easier to verify.

For system (1), if $\left\{i_{1}, i_{2}, \cdots, i_{m}\right\}=\{1,2, \cdots, m\}$ and $A_{i_{k+m}}=A_{i_{k}}, F_{i_{k+m}}(t, x)=F_{i_{k}}(t, x), k=$ 


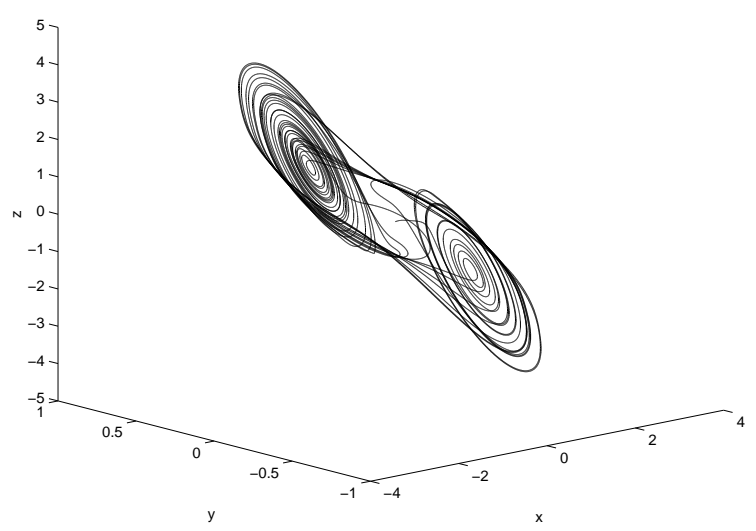

Fig. 1. Chaotic behavior of Chua's circuit

$1,2, \cdots$, then it is called a hybrid impulsive and periodic switching system, or system (1) has a periodic switching law. In this case, we have the further results.

Corollary 3.1. Assume that system (1) is a hybrid impulsive and periodic switching system with $t_{k}-$ $t_{k-1}=\tau_{k}, \tau_{k+m}=\tau_{k}, \beta_{k} \leq \beta, k=1,2, \cdots$, and $\lambda_{i}(t) \leq \lambda_{i}, i=1,2, \cdots, m, \tau_{k}, \beta$, and $\lambda_{i}$ are constants. Then

$$
m \ln (\rho \beta)+\lambda_{1} \tau_{1}+\cdots+\lambda_{m} \tau_{m}<0
$$

implies that the trivial solution of system (1) is globally asymptotical stable, where $\beta_{k}, \lambda_{i}(t)$, and $\rho$ are given by (7), (8), and (9), respectively.

\section{APPLICATION TO CHAOTIC CONTROL}

Consider Chua's circuit (Chua et al., 1986):

$$
\left\{\begin{array}{l}
\dot{x}_{1}=p\left(x_{2}-x_{1}-g\left(x_{1}\right)\right) \\
\dot{x}_{2}=x_{1}-x_{2}+x_{3} \\
\dot{x}_{3}=-q x_{2}
\end{array}\right.
$$

where $p>0, q>0, g\left(x_{1}\right)$ is a piecewise linear function given by

$$
g\left(x_{1}\right)=a x_{1}+\frac{1}{2}(b-a)\left(\left|x_{1}+1\right|-\left|x_{1}-1\right|\right),
$$

with $b<a<0$. The Chua's circuit is a typical chaotic system. Let $p=10, q=14.87, a=-0.68$, and $b=-1.27$, for instance, the system has a double scroll chaos attractor (Fig.1).

Rewrite system (23) as

$$
\dot{x}=A x+f(x),
$$

where $x=\left(x_{1}, x_{2}, x_{3}\right)^{\top}, f(x)=\left(-p g\left(x_{1}\right), 0,0\right)^{\top}$, and

$$
A=\left[\begin{array}{ccc}
-p & p & 0 \\
1 & -1 & 1 \\
0 & -q & 0
\end{array}\right]
$$

The controlled system can be described as

$$
\dot{x}=A x+f(x)+u(t, x)
$$

where $u(t, x)$ is the controlled input. We construct a hybrid impulsive and switching controller $u=$ $u_{1}+u_{2}$ for system (25) as follows:

$$
\begin{gathered}
u_{1}(t)=\sum_{k=1}^{\infty} B_{i_{k}} x(t) l_{k}(t), \\
u_{2}(t)=\sum_{k=1}^{\infty} B_{2 k} x(t) \delta\left(t-t_{k}\right),
\end{gathered}
$$

where $B_{i_{k}}$ and $B_{2 k}$ are $3 \times 3$ constant matrices, $B_{i_{k}} \in\left\{B_{1}, B_{2}, \cdots, B_{m}\right\}, \delta(\cdot)$ is the Dirac impulse, $l_{k}(t)=1$ as $t_{k-1}<t \leq t_{k}$, and otherwise, $l_{k}(t)=0$ with discontinuity points

$$
t_{1}<t_{2}<\cdots<t_{k}<\cdots, \lim _{k \rightarrow \infty} t_{k}=\infty,
$$

$t_{1}>t_{0}, t_{0} \geq 0$ is the initial time.

Moreover, it can be seen that, the hybrid impulsive and switching controlled system (25) has the following form:

$$
\begin{cases}\dot{x}=\left(A+B_{i_{k}}\right) x+f(x), & t \in\left(t_{k-1}, t_{k}\right] \\ \triangle x=B_{2 k} x, & t=t_{k} \\ x\left(t_{0}^{+}\right)=x_{0}, & k=1,2, \cdots,\end{cases}
$$

with $\triangle x\left(t_{k}\right)=x\left(t_{k}^{+}\right)-x\left(t_{k}\right)$.

For system (26), if $P_{i} \equiv I$, then $f^{\top}(x) x \leq$ $p|b| x^{\top} x$, that is, in $(6), \varphi_{i}(t)=p|b|, i=$ $1,2, \cdots, m$. Let $\tau=t_{k}-t_{k-1}$

$$
\begin{gathered}
\lambda_{\max }\left\{\left(A+B_{i}\right)^{\top}+\left(A+B_{i}\right)\right\}+2 p|b| \leq \lambda_{i}, \\
\lambda_{\max }\left\{\left(I+B_{2 k}\right)^{\top}\left(I+B_{2 k}\right)\right\} \leq \beta_{k},
\end{gathered}
$$

where $i=1,2, \cdots, m, k=1,2, \cdots$.

The corresponding results of Theorem 3.1, 3.2, and Corollary 3.1 easily follow.

For Chua's controlled system (26) with $p=10$, $q=14.87, a=-0.68$, and $b=-1.27$, if one chooses $m=2, B_{2 k}=\operatorname{diag}\{-0.86,-0.98,-0.78\}$,

$$
B_{1}=\left[\begin{array}{ccc}
9 & -16 & 0 \\
-22 & -30 & 0 \\
0 & 0 & 0
\end{array}\right], \quad B_{2}=\left[\begin{array}{ccc}
0 & 10 & 0 \\
-38 & 0 & 0 \\
0 & 0 & 0
\end{array}\right] \text {, }
$$

then it is easy to calculate and obtain

$$
\lambda_{1}=36.4824, \lambda_{2}=42.4678, \beta=0.0484 .
$$

If one takes $\tau=0.05$, then

$$
2 \ln (\beta)+\left(\lambda_{1}+\lambda_{2}\right) \tau=-2.1090<0,
$$

which implies, from Corollary 3.1, that the trivial solution of the controlled system (26), with the parameters $p=10, q=14.87, a=-0.68$, and $b=-1.27$, is asymptotically stable, that is, the Chua's circuit (23) is stabilized. The simulation result shown in Fig.2, indicates that the new hybrid impulsive and switching control strategy has many advantages, such as less time, less energy, 

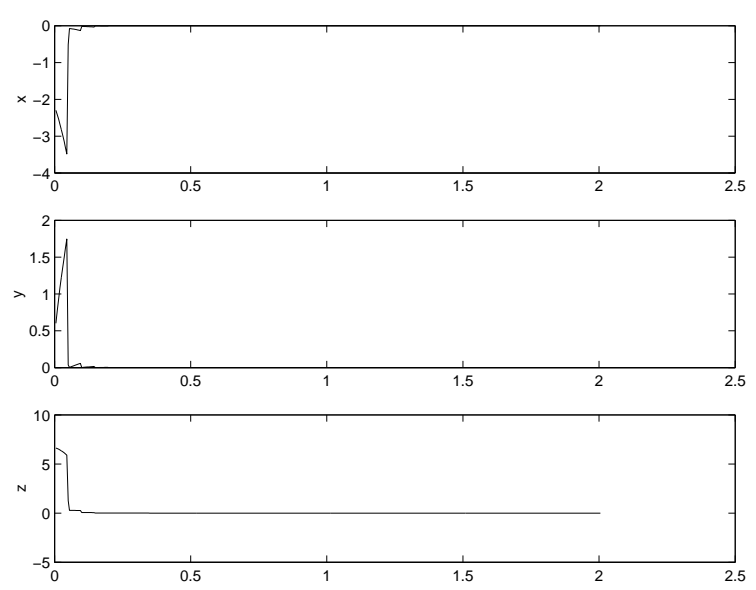

Fig. 2. The trajectories of the controlled system

and more flexible design, to achieve stabilization than the individual single continuous control, discrete control, impulsive control, or switching control in (Li et al., 2001).

\section{CONCLUSIONS}

In this paper, some new criteria for exponential stability and asymptotic stability of a class nonlinear hybrid impulsive and switching systems have been derived. Further more, a new hybrid impulsive and switching control strategy for nonlinear systems is developed. The research work should help to better understand the stability behavior of the nonlinear hybrid impulsive and switching systems.

\section{REFERENCES}

Aubin, J. P., J. Lygeros, M. Quincampoix, S. Sastry and N. Seube (2001). Impulse differential inclusions: A viability approach to hybrid systems. IEEE Trans. Automat. Cont. 47, 1-20.

Bainov, D. D. and P. S. Simeonov (1989). Stability Theory of Differential Equations with Impulse Effect: Theory and Applications. Ellis Horwood. Chichester.

Branicky, M. S. (1998). Multiple lyapunov functions and other analysis tools for switched and hybrid systems. IEEE Trans. on Automatic Contr. 43, 475-482.

Chua, L. O., M. Komuro and T. Matsumoto (1986). The double scroll family, part i: Rigorous proof of chaos. IEEE Trans. Circuits and Systems 33, 1073-1097.

Daafouz, J., P. Riedinger and C. Iung (2002). Stability analysis and control synthesis for switched systems: a switched lyapunov function approach. IEEE Trans. Automat. Contr. 47, 1883-1887.
Decarlo, R. A., M. S. Branicky, S. Pettersson and B. Lennartson (2000). Perspective and results on the stability and stabilizability of hybrid systems. Proc. IEEE 88, 1069-1082.

Ge, S. S., Z. Sun and T. H. Lee (2001). On reachability and stabilization of switched linear discrete-time systems. IEEE Trans. Automat. Contr. 46, 1437-1441.

Guan, Z. H., G. Chen and T. Ueta (2000). On impulsive control of a periodically forced chaotic pendulum system. IEEE Trans. Automat. Contr. 45, 1724-1727.

Hespanha, J. P. and A. S. Morse (1999). Satbility of switched systems with average dwell-time. in Proc. 38th Conf. Decision and Control 2655-2660.

Ishii, H. and B. A. Francis (2002). Stabilizing a linear system by switching control with dwell time. IEEE Trans. Automat. Contr. 47, 1962-1973.

Khadra, A., X. Liu and X. Shen (2003). Application of impulsive synchronization to communication security. IEEE Trans. on Circuits and Systems 50, 341-350.

Lakshmikantham, V., D. D. Bainov and P. S. Simeonov (1999). Theory of Impulse Differential Equations. World Scientific. Singapore.

Leonessa, A., W. M. Haddad and V. Chellaboina (2001). Nonlinear system stabilization via hierarchical switching control. IEEE Trans. Automat. Contr. 46, 17-28.

Li, Z. G., C. Y. Wen, Y. C. Soh and W. X. Xie (2001). The stabilization and synchronization of Chau's oscillators via impulsive control. IEEE Trans. Circuit. Syst. I 48, 1351-1355.

Liberzon, D. (2003). Switching in Systems and Control. Birkhauser. Boston.

Liberzon, D. and A. S. Morse (1999). Basic problems in stability and design of switched systems. IEEE Control Systems Magazine 19, 59-70.

Liu, Y. Q. and Z.H. Guan (1996). Stability, Stabilization and Control of Measure Large-Scale Systems with Impulses. The South China University of Technology Press. Guangzhou.

Mancilla-Aguilar, J. L. (2003). A condition for the stability of switched nonlinear systems. IEEE Trans. Automat. Contr. 45, 2077-2079.

Michel, A. N. (1999). Recent trends in the stability analysis of hybrid dynamical systems. IEEE Trans. Circuit. Syst. 46, 120-134. 